\title{
Cold Model Study of Emulsification Behavior in Bottom Blown Metallurgical Baths Covered with Thick Slag
}

\author{
Leili Tafaghodi KHAJAVI and Mansoor BARATI \\ Department of Materials Science and Engineering, University of Toronto, 184 College Street, Toronto, ON, M5S 3E4 Canada. \\ E-mail: leili.tafaghodikhajavi@utoronto.ca
}

(Received on November 6, 2009; accepted on February 27, 2010)

\begin{abstract}
In the processes with two interacting immiscible liquids, the overall rate of the inter-phase reactions is typically controlled by chemical reaction at the interface, mass transport within each phase, or combination of both. Under these conditions, the overall rate of the reaction between the two phases is closely related to the behaviour of their interface, the interfacial area in particular. In this work, aiming to study emulsification and the associated interfacial area increase in bottom blown metallurgical systems, water modeling was used. For two different water-oil systems, the droplet size distribution, water-oil interfacial area increase, and their dependence on gas flow rate and physical properties of the liquids were studied. The total interfacial energy and the fractions dissipated in forms of surface and potential energy were calculated.
\end{abstract}

KEY WORDS: emulsification; droplet; interfacial area; water modeling; interfacial energy.

\section{Introduction}

Bath mixing by means of gas injection is one of the most routine practices in many chemical and metallurgical processes. Interphase mixing in forms of droplet generation or emulsification, is a characteristic of the systems containing two immiscible liquids such as slag and metal/matte. For examples, in bottom blown bath smelting of iron, ${ }^{1-3)}$ iron droplets form in the slag and increasing the gas flow rate intensifies the droplet suspension. Also, it is well known that the ejection of metal droplets to slag in Basic Oxygen steelmaking accelerates the refining reactions considerably.

Several experimental and mathematical studies ${ }^{4-13)}$ have been carried out on slag/metal droplet generation in the systems agitated by gas injection. The mechanism of droplet generation and emulsification have been investigated through low temperature water modeling ${ }^{4-6,14-16)}$ as well as high temperature studies of slag-metal interactions. ${ }^{1,2,5,8-10)}$ For example, dispersion of paraffin oil in water and mercury in paraffin oil have been reported by Tanaka and Guthrie. ${ }^{14)}$ In separate works on mercury/water-glycerin and mercury/alcohol systems, ${ }^{15,16)}$ it has been found that a film of the lower liquid on the bubble surface is carried to the upper phase and when the bubble bursts, many small droplets are generated. Zaidi and Sohn ${ }^{4)}$ suggested a correlation between the metal droplets size distribution and appropriate dimensionless numbers characterizing the operating conditions. In high gas flow rate, beyond a critical value, the turbulence at the interface brings about entrainment of slag droplets or ligaments in the metal ${ }^{17,18)}$ in a phenomenon known as inverse emulsification. The accelerated rate of reactions at high gas flow rate has been attrib- uted to this phenomenon in some cases. ${ }^{19,20)}$ On the other hand, Dayal and coworkers ${ }^{5,7)}$ found small metal droplets in the bulk of the slag, while no slag droplet has been detected in the metal bath.

It is well accepted that emulsification of any nature contributes to the rate of the reactions and the mass transfer between slag and metal. The rate of mass transport between the two phases is directly related to interfacial area, and is expressed as:

$$
\stackrel{\circ}{m}=k A\left(C_{b}-C_{1}\right) \text {. }
$$

where $k$ is the mass transfer coefficient, $A$ is the interfacial area and $\left(C_{b}-C_{1}\right)$ represents the concentration gradient across the interface.

In order to predict the mass transfer kinetics, one has to quantify the interfacial area. However, due to experimental difficulties, little has been achieved in terms of direct measurements of the interfacial area created by the dispersed droplets. Sample withdrawal methods such as the suction pipe technique ${ }^{21)}$ have been widely used to quantify droplet size and distribution. However, most of the methods are subject to significant errors if great care is not taken in sampling because of possibility of droplet breakup, coalescence or preferential sampling. In addition, in order to obtain meaningful results, and due to stochastic nature of the phenomenon, a large number of droplets (and samples) has to be withdrawn so that reliable averaging can be performed. This is practically limited by discontinuous measurement methods such as the suction pipe technique.

Very often, the rate of mass transport of tracer species between two immiscible liquids has been measured as an indication of the interfacial area in the emulsion. Most of the studies in this area have obtained information about an 
"apparent" or volumetric mass transfer coefficient, sometimes referred to as mass transfer parameter, ${ }^{22)}$ which is equivalent to $k A$ in Eq. (1). There are several studies of mass transfer rate in cold systems such as water/oil, ${ }^{22-24)}$ water/benzene, ${ }^{25)}$ water/clyclohexane, ${ }^{26)}$ mercury/aqueous and amalgam/aqueous, ${ }^{27-30)}$ and melts such as lead/molten salt. ${ }^{30,31)}$ Considerable amount of knowledge has been accumulated on the overall mass transfer rate between two immiscible liquids, by generally monitoring the transport of a tracer with a specific partition ratio between the two liquids. Some investigators have attempted to avoid interfacial turbulence, thus by assuming a planner interface they were able to calculate the intrinsic mass transfer coefficient. In the earlier works carried out by Richardson and his group, ${ }^{27,28,30)}$ the mass transfer coefficient was obtained by using a constant interfacial area. They found that the mass transfer coefficient is proportional to the square root of $Q / A$ where $Q$ is the gas flow rate and $A$ is the cross section (planner interface area) of the container. They further showed that the mass transfer rates are independent of the method of gas introduction (i.e. single or multiple streams). Patel et $a l .{ }^{32)}$ on the other hand found that the mass transfer coefficient in bubble-stirred aqueous-organic system is proportional to $Q^{0.72}$. Asai et $a l .^{33)}$ have obtained a range of exponent for $Q$ from 0.25 to 3.0, by analyzing the results of previous works in this area.

In a similar fashion to the mass transfer studies and using thermal tracer, Sohn et al. ${ }^{34)}$ indicated that the overall heat transfer between the two liquids in gas agitated TCE/Water and Kerosene/Water systems is proportional to the energy input rate per unit volume of the liquid.

Generally, the interfacial reaction area created by bottom blowing cannot be deduced from the measured transport rate because in different gas flow rate, the velocity of droplets, and consequently the mass transport coefficient (k) are rather variable. Therefore, the mass transfer coefficient and the interfacial area cannot be separated out from the overall mass transfer rate $k A$. One has to quantify either the mass transfer coefficient or the interfacial area to calculate the other. This research aims at direct measurement of the droplet size distribution in different emulsions to quantify the interfacial area between the two liquids. In particular, the emulsification behavior and interfacial area increase associated with the presence of a thick oil layer which has attracted much less attention in previous researches is studied.

\section{Experimental Works}

A water bath covered with a layer of oil to represent slag was used for the experiments. The liquids were contained in a cylindrical vessel with diameter of $33 \mathrm{~cm}$. Schematic of the setup used for monitoring interphase mixing is depicted in Fig. 1. In all experiments, the bath is agitated by injecting compressed air through a single bore nozzle located at the center bottom of the tank. The diameter of the nozzle is $2 \mathrm{~mm}$. The properties of the two types of oil that were used as the upper phase are provided in Table $\mathbf{1 .}$

The purpose of this study was to quantify the interfacial area in the emulsified region. Specifically it was desired to obtain information on the volume and size of the oil

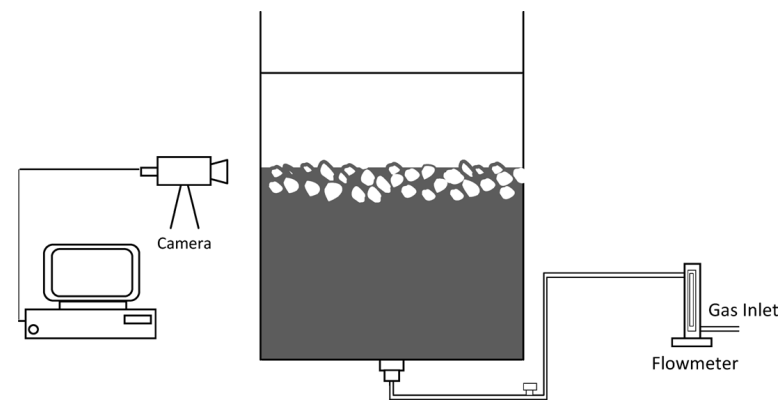

Fig. 1. Schematic of the equipment used to simulate bath mixing with overlaying layer.

Table 1. Physical properties of kerosene and silicone oil.

\begin{tabular}{llll}
\hline Oil type & Density $\left(\mathrm{gr} / \mathrm{cm}^{3}\right)$ & Viscosity $(\mathrm{cSt})$ & $\begin{array}{l}\text { Interfacial tension } \\
\text { with water }(\mathrm{dyne} / \mathrm{cm})\end{array}$ \\
\hline Kerosene & 0.79 & 1.14 & 58.2 \\
Silicone Oil & 0.96 & 50 & 63.9 \\
\hline
\end{tabular}

droplets. Interfacial area in the emulsified region can then be calculated based on these measurements. The method to quantify the volume of the droplets involves detecting the volume change of the emulsion when gas blowing is stopped. The volume change is measured by analyzing the images that are taken using a high speed CCD camera. The change in the volume of the emulsion is related to the separation of droplets from their original phase. Using this simple measurement, the total volume of entrained droplets, $V_{d}$ can be estimated. In order to obtain the droplet size distribution, several still images taken during the experiment and after the steady state is reached, are studied by an image analysis package. The data collected over the measurement time are combined to produce a set of droplet size distribution data, based on which, the Sauter mean diameter can be calculated. Two types of images were taken for each experimental condition. The first type is 5 to 8 high magnification images from different parts of the vessel, these images were analyzed to obtain the size distribution of the droplets. Depending on the volume of the emulsified region, the area $\left(\mathrm{mm}^{2}\right)$ of 50 to 170 droplets were measured for each experimental condition. The second type of image has lower magnification and shows the entire emulsified region from which the thickness of the emulsified region is measured. The height of this layer is calculated by averaging the measured values in 5 to 15 different places around the vessel periphery. These values are also directly measured by a digital caliper. The values obtained from image analysis are confirmed by comparing with the values acquired with the digital caliper.

The cylinder wall has a curvature that introduces a small error in the measured droplets area, due to stretching of the image in the horizontal direction. This was corrected by taking five images of a small marble submerged in the water bath at the same place where images for droplet size measurement were taken. Comparing the size of the marble obtained from the images with the actual marble size, a correction factor of 0.98 was applied for droplet size.

Two series of experiments were conducted in the same vessel, with water being the lower phase, and kerosene and silicone oil as the overlaying slag layer, respectively. Water height was kept constant at $19.8 \mathrm{~cm}$ during these experi- 
ments, while the oil height varied from 3.3 to $16.4 \mathrm{~cm}$ with equal intervals of $3.3 \mathrm{~cm}$. The air flow rate varies from 2.13 to $13.12 \mathrm{lpm}$ for all oil heights.

\section{Results and Discussion}

Measuring the size distribution of the dispersed droplets, the Sauter mean diameter $(\tilde{d})$ and the interfacial area $\left(A_{i}\right)$ can be calculated from the following equations, assuming that the droplets are spherical.

$$
\begin{gathered}
\tilde{d}=\frac{\sum d_{i}^{3}}{\sum d_{i}^{2}} \\
A_{i}=\frac{6 V_{d}}{\tilde{d}}
\end{gathered}
$$

where $d_{i}$ is the equivalent diameter of a single droplet and $V_{d}$ is the volume of the entrained droplets.

An image of the droplets which are formed in the presence of a $6.6 \mathrm{~cm}$ thick silicone oil layer with the air flow rate of $2.13 \mathrm{lpm}$ is shown in Fig. 2.

\subsection{Determination of the Nature of Emulsified Droplets}

Dispersion of metal in slag is a well known phenomenon in gas stirred metallurgical vessels. Depending on the operational variables such as gas flow rate, upper phase thickness and physical properties of the liquids, slagdroplets may also be present in the metal. As previously reported $^{22,26)}$ entrainment of slag in metal may occur and give rise to an increased mass transfer rate between slag and metal. In order to identify the dominant type of emulsification, i.e. direct (water droplets in oil) or inverse (oil droplets in water), a series of simple measurements and calculations were conducted. Figure 3 shows different layers of a bath after the formation of emulsified layer. $h_{m}{ }^{\prime}$ and $h_{s}{ }^{\prime}$ are respectively water and oil thicknesses after the emulsified layer is formed. As it is depicted, the emulsified region is extended on both sides of the original interface between oil and water. The emulsified region is divided into two hypothetical parts. Since the volume of the bath content (and subsequently the total bath height) does not change after emulsification, the contribution of each phase to the volume of the emulsified region should be equal to its volume change before and after emulsification. In other words the volumes of entrained water and oil within the emulsified region are equal to $\left(h_{m}-h_{m}{ }^{\prime}\right) \pi R^{2}$ and $\left(h_{s}-h_{s}{ }^{\prime}\right) \pi R^{2}$ respectively, where $R$ is the radius of the vessel.

The initial heights of water $\left(h_{m}\right)$ and $\left(h_{s}\right)$ oil layers are known for each experiment. $h_{e m}$ and $h_{s}{ }^{\prime}$ are measured from the low magnification images. Evidently, by knowing the values of $h_{s}$ and $h_{s}{ }^{\prime}, h_{e m-s}$ and $h_{e m-m}$ can be calculated from Eqs. (4) and (5) respectively.

$$
\begin{gathered}
h_{e m-s}=h_{s}-h_{s}{ }^{\prime} \\
h_{e m-m}=h_{e m}-h_{e m}
\end{gathered}
$$

Calculating the values of $h_{e m-s}$ and $h_{e m-m}$ provides the fractions of the emulsified region which are occupied by

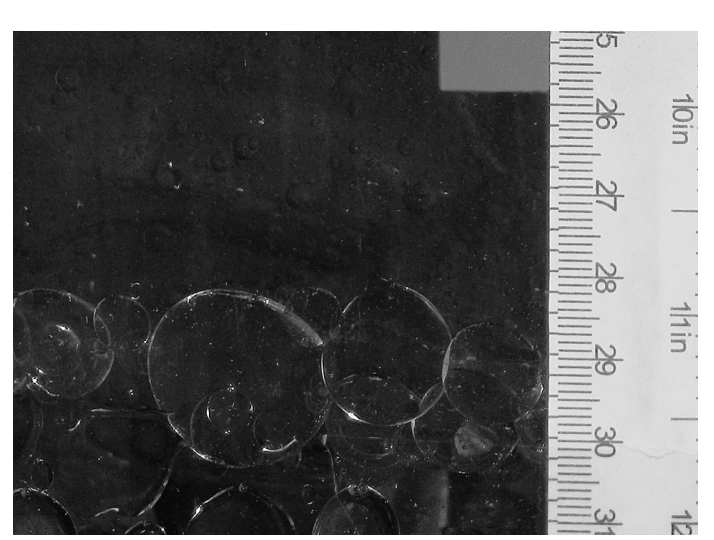

Fig. 2. Droplets formed at the presence of a $6.6 \mathrm{~cm}$ thick silicone oil layer with the air flow rate of $2.13 \mathrm{lpm}$.

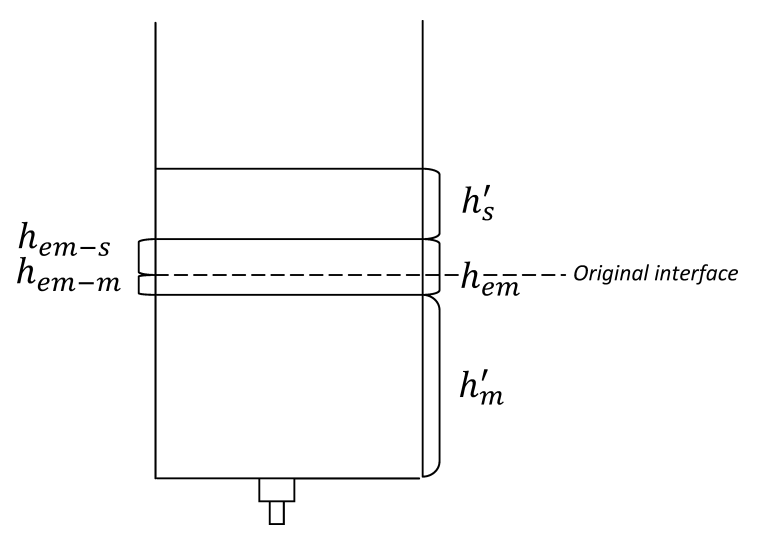

Fig. 3. Schematic of the emulsified region formed between oil and water.

each phase. In most experimental conditions, the upper oil phase fills over 50\% (43-92\%) of the emulsified region. By analyzing high magnification images, it is estimated that 55 to $84 \%$ of the volume of the emulsified region is occupied by droplets and the remaining is the second phase, surrounding them. This estimation is based on the assumption that the distribution of the droplets in the 2-dimensional image taken from the side of the vessel represents the distribution thereof in the bulk. In most cases the difference between the fraction of droplets obtained from the two liquid thicknesses and the fraction obtained from image analysis is less than $10 \%$. As a result it is reasonable to conclude that the droplets and the surrounding phase are oil and water respectively. Assuming that the total volume of entrained droplets in emulsified region, $V_{d}$, is equal to the volume of oil in the emulsified region, it can be calculated from Eq. (6).

$$
V_{d}=\pi R^{2}\left(h_{s}-h_{s}{ }^{\prime}\right)
$$

\subsection{Size Distribution of the Droplets in Emulsion}

As previously discussed, any two phase reaction is considerably promoted by gas stirring, as a result of fast replacement of the lower phase and also increasing the interfacial area. Interfacial area of the emulsified region is calculated by substituting the value of total volume of entrained droplets in emulsified region $\left(V_{d}\right)$ - obtained from Eq. (6) - back into Eq. (3). The Sauter mean diameter (SMD) of the droplets and the associated coefficient of 

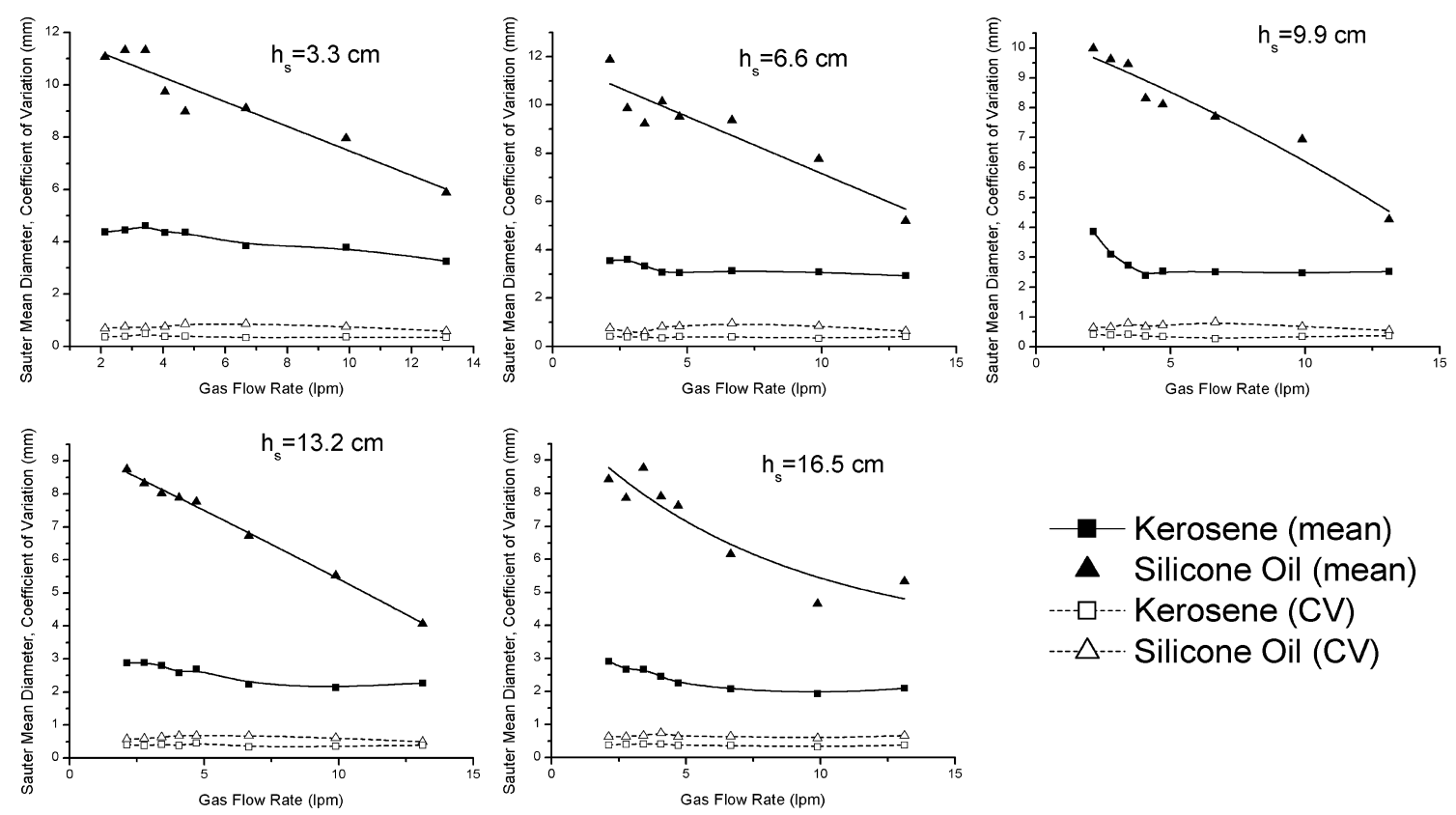

Fig. 4. Sauter mean diameter and coefficient of variation of the droplets size distribution at various experimental conditions.

variation are plotted against gas flow rate in Fig. 4. The larger SMD of the droplets in water/silicone oil system may be attributed to higher viscosity of silicone oil, which results in larger shear stress at the interface for a given velocity gradient. This greater shear stress can overcome a larger buoyancy force that acts against droplet detachment. In other words, droplets with greater buoyancy, i.e. larger size, can be formed in water-silicone oil system. As a general trend, the SMD of the droplets in both water-kerosene and water-silicone oil baths decrease with gas flow rate. Increasing gas flow rate results in highly unstable water/oil interface at the edge of the uprising plume and the upper phase. The increased recirculation velocity intensifies the impact of the bubbling. Consequently, the resulting downward shear flow on both oil ligaments and previously formed droplets increases, leading to formation of smaller droplets at higher gas flow rate.

As seen in Fig. 4, increasing gas flow rate decreases SMD in the water-silicone oil systems, whereas in the water-kerosene baths, this trend is not noticeable at higher flow rates and starts to level off at around $5 \mathrm{lpm}$. The difference between the relationship of SMD and gas flow rate in water-kerosene and water-silicone oil baths is explained as follows. When a droplet experiences the sheer stress of the adjacent moving fluid, it can remain stagnant or be pushed aside, depending on its inertia and the magnitude of the stress. Larger droplets will be more difficult to be pushed away, thus they are more likely to break into small droplets. In contrast, for droplets with less inertia, i.e. smaller droplets, the velocity at the interface moves the droplet around instead of breaking it. The density and size of silicone oil droplets are greater than those of kerosene, thus they can be more readily split into smaller droplets by increasing the gas flow rate. It is reasonable to consider that for a specific system of liquids and velocity profile, there is a critical droplet size below which breakage of droplets cannot take place. This is consistent with the results shown in Fig. 4, indicating that in water-kerosene baths average droplet size cannot be smaller than $2-4 \mathrm{~mm}$ even at high flow rates.

Residence time of the droplets in the emulsified region also has a substantial influence on the size distribution of the droplets in the emulsion. After formation of an oil droplet in the lower phase, the net upward force on the generated droplet is the difference between its gravity and buoyancy. Obviously smaller upward force results in slower return of the droplet to the upper phase and longer residence time. As the density difference in water-silicone oil baths, $\left(0.04 \mathrm{~g} / \mathrm{cm}^{3}\right)$, is smaller than that of water-kerosene baths $\left(0.21 \mathrm{~g} / \mathrm{cm}^{3}\right)$, the residence time of silicone droplets is longer than kerosene droplets. On the other hand, the residence time of the large droplets is shorter than that of the small ones, so more large droplets return to upper phase rather than small droplets. The density difference affects the residence time of the large droplets to a greater extent than that of the small ones, thus the fraction of the larger droplets remaining in emulsion is more in water-silicone oil baths. In other words, classifying the droplets into two "large" and "small" categories, the fraction of the so called large droplets that remain in emulsion is more in water-silicone oil baths compared to the water-kerosene system. It results in greater SMD in water-silicone oil baths. As shown in Fig. 4, the coefficient of variation is also greater in water-silicone oil baths compared to water-kerosene. This observation is consistent with the above explanation, as the further breakage of large droplets creates a wider spread in their size. Also, in water-kerosene systems, droplets essentially reach the smallest possible (most stable) size, thus the size distribution is narrower. Distribution of the droplet size in the emulsified layer of water-kerosene and water-silicone oil baths are shown in Figs. 5 and 6 respectively. 
ISIJ International, Vol. 50 (2010), No. 5
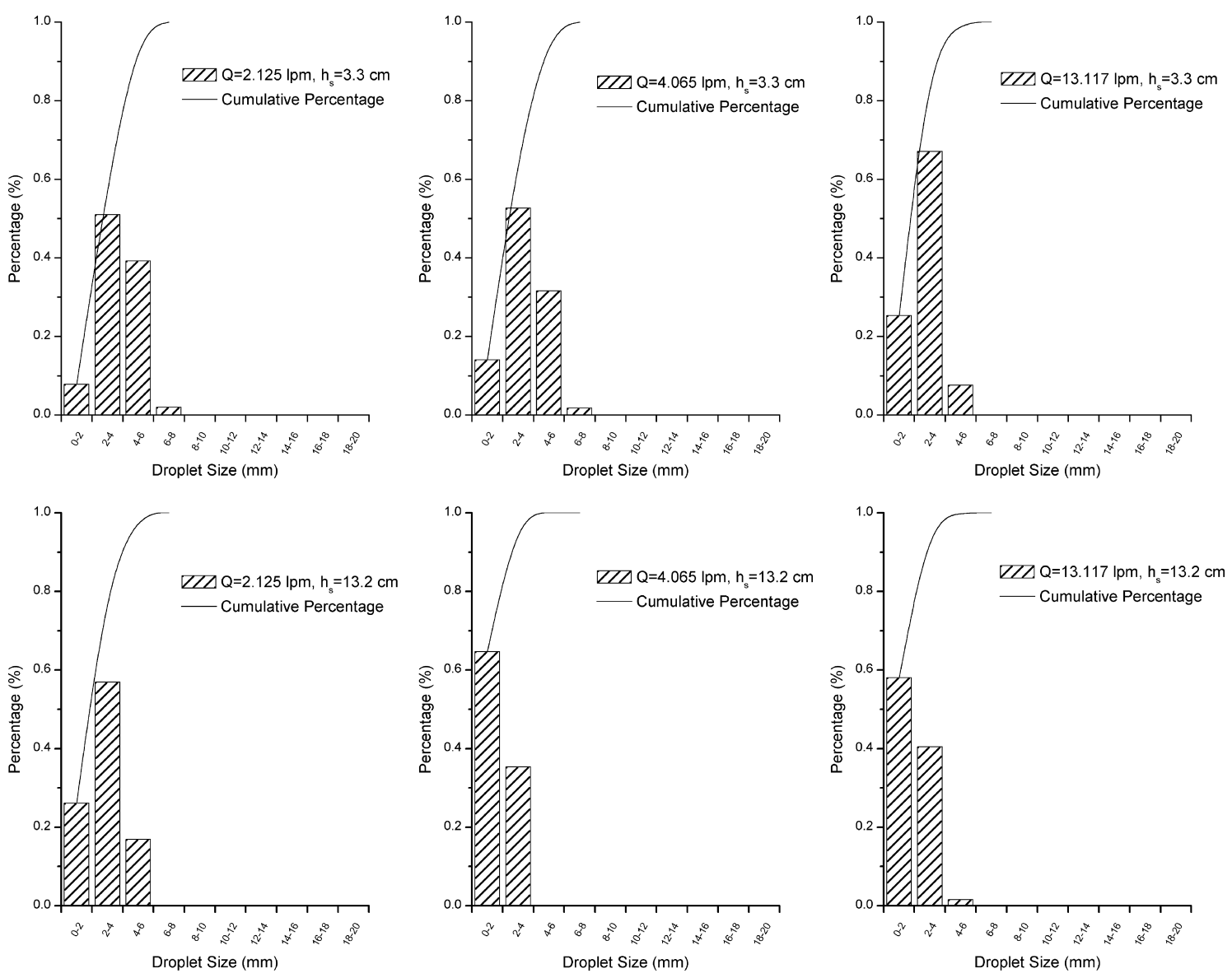

Fig. 5. Sample size distributions of the droplets in the emulsified region of water-kerosene baths.
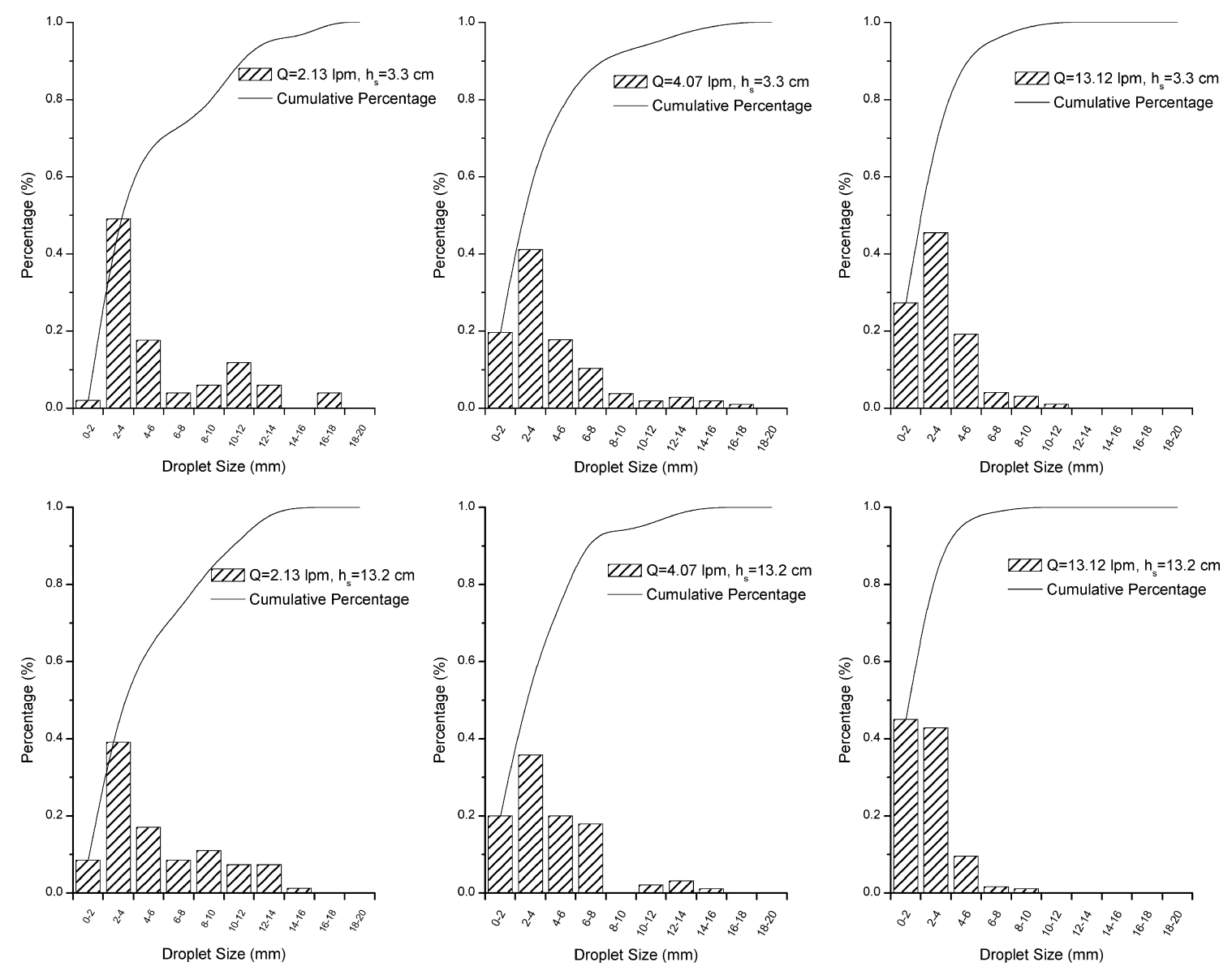

Fig. 6. Sample size distributions of the droplets in the emulsified region of water-silicone oil baths. 


\subsection{Dependence of Emulsification Behavior on the Fluids Properties}

The difference between physical properties of kerosene and silicone oil can lead to different responses of the two systems to bath agitation. It is well accepted that higher oil density facilitates formation of the droplets, because of lower upward force (net difference between gravity and buoyancy) acting against it during and after detachment from the interface. The same effect is expected with decreasing interfacial tension $(\sigma)$ between the two fluids. The higher the interfacial tension, the more the interfacial energy required for formation of a droplet of given size. Weber number is used to analyze fluid flow where there is an interface between two different fluids. It is particularly used as a measure of interface instability for multi-phase flows with strongly curved surfaces, such as droplets and bubbles, ${ }^{35)}$ and is expressed as:

$$
W e=\frac{\rho L u^{2}}{\sigma}
$$

where $\rho$ is the density of the fluid, $u$ is its velocity, $L$ is the characteristic length, typically being the droplet diameter and $\sigma$ is the interfacial tension between the two liquids. As shown in Table 1, the interfacial tension between waterkerosene and water-silicone oil are $58.2 \mathrm{dyn} / \mathrm{cm}$ and 63.9 dyne/cm, respectively. The density differences between the two liquid phases are $0.21 \mathrm{~g} / \mathrm{cm}^{3}$ and $0.04 \mathrm{~g} / \mathrm{cm}^{3}$ for waterkerosene and water-silicone oil baths. As seen in Fig. 4, in water-kerosene and water-silicone oil system with the oil height equal to $3.3 \mathrm{~cm}$ and gas flow rate of $2.131 \mathrm{pm}$ the Sauter mean diameter of the droplets are 4.38 and $11.08 \mathrm{~mm}$ respectively. The Weber number for the two systems under these conditions can thus be estimated as:

$$
\begin{gathered}
W e_{\text {kerosene }}=0.0059 u^{2} \ldots \\
W e_{\text {silicone oil }}=0.0166 u^{2} .
\end{gathered}
$$

Similar equations can be obtained for other experimental conditions, i.e. different slag heights and gas flow rates by using the SMD acquired for each condition. The coefficients of Eqs. (8) and (9) vary between 0.0029-0.0059 and $0.0080-0.0166$ respectively, while it always has a greater value in water-silicone oil baths. Equations (8) and (9) can be used to discuss the difference in the emulsification behaviours of water-kerosene and water-silicone oil systems. According to these equations, a larger emulsified region in water-silicone oil baths may be attributed to a less stable interface, i.e., larger Weber number, for a given velocity profile. Similar justification can be made for further emulsification and easier breakage of the droplets that was observed in water-silicon oil system.

\subsection{Analysis of Interfacial Area in Emulsified Region}

Figure 7 shows a plot of interfacial area in the emulsified region as a function of gas flow rates for different thicknesses of kerosene. As seen, in the log-log scale, the interfacial area increases almost linearly with increase in the gas flow rate. It is also clear that the interfacial area is larger for baths containing a thicker oil layer. This can be related to

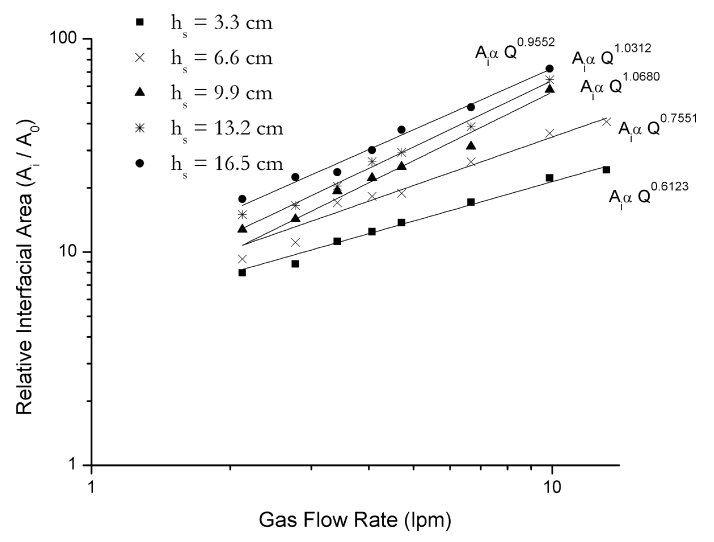

Fig. 7. Interfacial area of the emulsified region at the presence of kerosene as the upper phase.

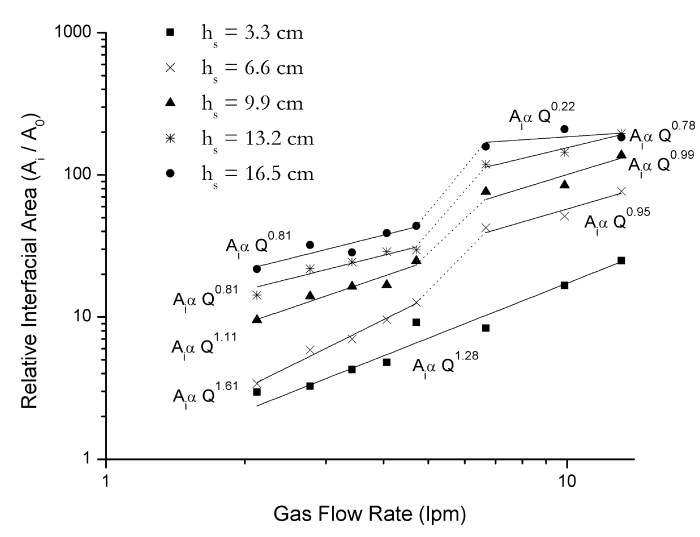

Fig. 8. Interfacial area of the emulsified region at the presence of silicone oil as the upper phase.

the fact that for a thicker upper phase, the pressure at the interface between oil and water is larger. This facilitates separation of a droplet from oil into water. Deeper oil also increases the height of the emulsified region which leads to longer residence time of the droplets in the emulsion.

Figure 8 shows interfacial area of the emulsified region as a function of gas flow rates for different thicknesses of silicone oil. Similar to water-kerosene baths, interfacial area increases with gas flow rate and oil thickness. Significant increase in the interfacial area up to two orders of magnitude was observed under some experimental conditions such as gas flow rates higher than $6.66 \mathrm{lpm}$ and oil thicknesses greater than $13.2 \mathrm{~cm}$. However, an abrupt increase of $A_{i}$ is seen when the gas flow rate is increased from 4.71 to $6.66 \mathrm{lpm}$. The results are consistent with the observations that when the gas flow rate exceeds this critical value, the entire oil layer breaks into small droplets, whereas at smaller flow rates, only a certain thickness of the layer is emulsified. The abrupt increase in the area was not seen when silicone oil thickness was $3.3 \mathrm{~cm}$, because the oil layer did not break entirely into droplets for this thickness. It is also noticeable that for each oil height, the slope of the second section of the graph is lower than the slope of the first part. It is expected to see this trend because by increasing the flow rate to the point where the entire oil layer is emulsified, a state is reached where further increase of flow rate can only break large droplets into smaller ones. This stage has a less pronounced effect on the interfacial area compared to the case where the liquid bulk 
is divided to numerous droplets. The sudden increase of $A_{i}$ does not occur in water-kerosene experiments because only a fraction of the kerosene layer breaks into droplets at the experimental conditions mentioned in Chap. 2. In other words, in water-kerosene systems a fraction of the oil remained as a separate layer and did not enter the emulsified region. This is consistent with the Weber number criteria discussed earlier. Smaller value of Weber number in water-kerosene baths implies that emulsification is more difficult compared to water-silicone oil baths.

\subsection{Estimation of the Mass Transfer Coefficient}

Independent estimation of the interfacial area, $A_{i}$, would enable one to derive $k$ from the apparent mass transfer rate constant. As an example, the values of $k$ in Kim and Fruehan's study ${ }^{22}$ are discussed here. They used water and a $50 / 50$ mixture (by volume) of paraffin oil and cottonseed oil as upper and lower phases, respectively. Mass transfer measurements were made for benzoic acid rod dissolving into water. They assumed that $k$ is the same in both experiments. In their work $k A_{i}$ was measured based on following equation.

$$
\frac{d W}{d t}=k A\left(C_{i}-C_{b}\right)
$$

where $C_{i}$ and $C_{b}$ are concentrations at interface and bulk respectively, $d W / d t$ is the weight loss of the rod within a specific time. $A$ is the exposed area of the benzoic acid rod in water and was assumed to be the average of the initial and final area of the tip of the rod.

It is possible to estimate interfacial area in Kim and Fruehan $^{22)}$ experimental conditions using the obtained trend in Fig. 8. Although the physical properties of the liquids used in their experiment $\left(\rho=0.896 \mathrm{~g} / \mathrm{cm}^{3}, \sigma=19.38 \mathrm{dyn} /\right.$ $\mathrm{cm}, \nu=53 \mathrm{cst}$ ) are not exactly similar to that of this study, the silicone oil physical properties, especially its high viscosity, that dominantly controls the mass transfer coefficient, are reasonably close to the properties of silicone oil used in this study. The interfacial area at the presence of a $3.3 \mathrm{~cm}$ thick silicone oil layer, for gas flow rates of 2.72 , 3.70 and $4.95 \mathrm{lpm}$, are estimated to be 2979,4275 and $6131 \mathrm{~cm}^{2}$ respectively. Dividing the $k A$ values estimated by Kim and Fruehan by these numbers, the mass transfer coefficients for the above conditions are calculated as $2.03 \times$ $10^{-3}, 2.1 \times 10^{-3}$ and $4.4 \times 10^{-3} \mathrm{~cm} / \mathrm{s}$ respectively. The values of $k$ estimated by Kim and Fruehan are $1.05 \times 10^{-3}$, $1.25 \times 10^{-3}$ and $1.55 \times 10^{-3} \mathrm{~cm} / \mathrm{s}$ respectively. It can be seen that the values obtained by them are 2 to 3 times smaller than those of current study. This is considered good agreement, noting that the physical properties of their fluid are different fromthe ones used in present work, and also their estimated interfacial area is very approximate.

\subsection{Energy Dissipation Associated with Interfacial Phenomena of Emulsified Region}

Energy dissipation associated with emulsions is composed of two components: the surface energy required for the formation of droplets, $E_{s, d}$ and the potential energy, $E_{p, d}$ required to maintain the droplets in the other phase. The energy consumed in the first part can be estimated by multiplying the total area of the droplets by the interfacial ten-

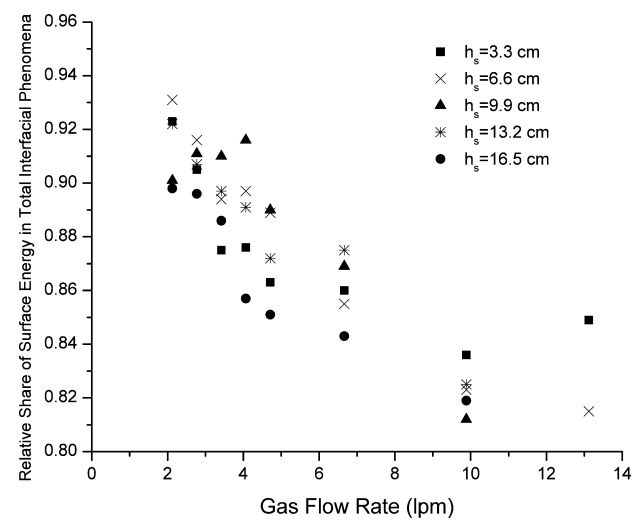

Fig. 9. Relative share of surface energy associated with formation of droplets at the presence of kerosene as upper phase.

sion between the two liquid phases.

$$
E_{s, d}=A_{i} \sigma \ldots
$$

In order to obtain the second component, the applied force on the droplets should be calculated. This force is equal to the difference between the gravity and buoyancy. Assuming that the average displacement of oil droplets is equal to half of the emulsified layer thickness, the potential energy for maintaining the oil droplets in water can be calculated from Eq. (12).

$$
E_{p, d}=\left(\rho_{m}-\rho_{s}\right) V_{d} g \frac{h_{e m}}{2}
$$

The total energy associated with interfacial phenomena of oil droplets in emulsified region, $E_{i, d}$ is thus calculated from Eq. (13).

$$
E_{i, d}=A_{i} \sigma+\left(\rho_{m}-\rho_{s}\right) V_{d} g \frac{h_{e m}}{2}
$$

Figure 9 shows the relative share of surface energy required for formation of droplets in emulsified region in the presence of kerosene as upper phase. The contribution of surface energy to total interfacial energy is between 80 to $95 \%$ in water-kerosene baths. Although the absolute values of surface energy increases with gas flow rate and oil thickness, its relative portion in the total interfacial energy decreases with both gas flow rate and oil height. This trend is expected based on the discussion in Sec. 3.2 about the breakage of large droplets into smaller ones by increasing the gas flow rate. Breakage of large droplets into smaller ones becomes more significant by increasing gas flow rate. This breakage has a less noticeable effect on the interfacial area than the effect of formation of droplets from bulk. Consequently, surface energy needed for generating this area is less affected at high flow rates. By increasing gas flow rate, the contribution of breakage of droplets in total surface generation becomes more, consequently the proportional value of surface energy decreases and it becomes relatively constant at the end.

A similar pattern is seen in Fig. 10 which shows the relative share of surface energy required for formation of droplets in emulsified region at the presence of silicone oil as upper phase. The percentage of surface energy to total interfacial energy is between 55 to $95 \%$ in water-silicone 


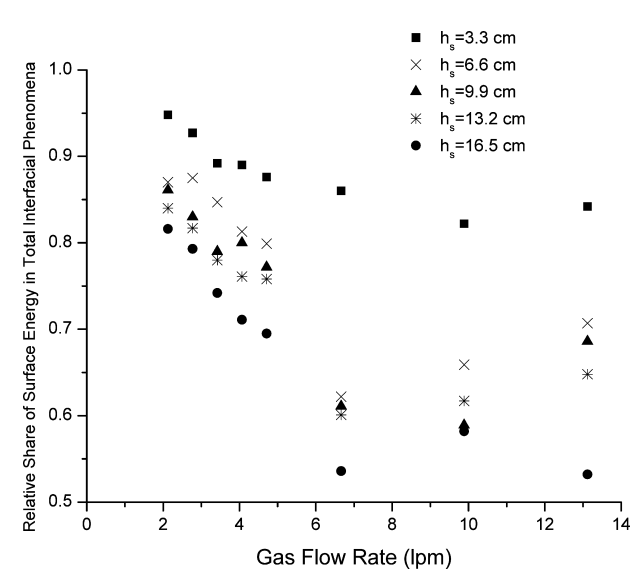

Fig. 10. Relative share of surface energy associated with formation of droplets at the presence of silicone oil as upper phase.

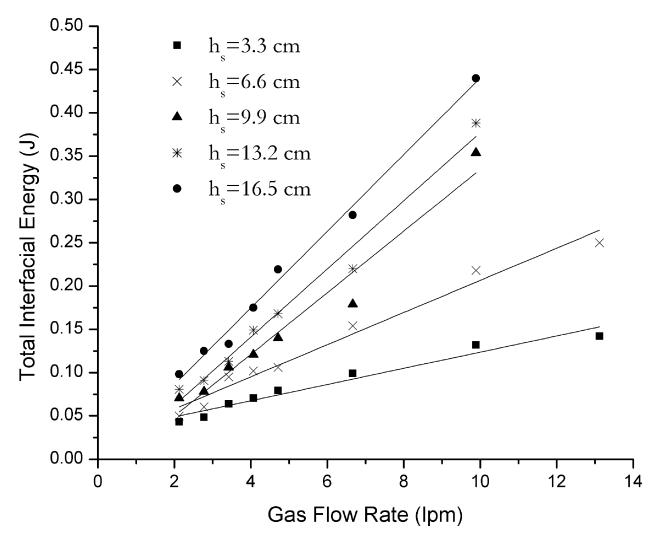

Fig. 11. Interfacial energy associated with the emulsified region at the presence of kerosene as upper phase.

baths. The difference between this relationship in waterkerosene and water-silicone oil baths is that the relative share of surface energy in water-silicone oil baths increases or becomes relatively constant at around $6.66 \mathrm{lpm}$. This critical flow rate is consistent with the flow rate at which the abrupt change in interfacial area took place. At this stage further increase of flow rate can only break large droplets into smaller ones, indicating that the entrainment of new droplets becomes negligible so the volume of entrained oil droplets and the emulsified height are approximately constant, but the interfacial area is increased. In this case the potential energy is relatively constant while the surface energy increases as a result of droplet breakage.

Figure 11 shows the total interfacial energy against gas flow rate for different oil heights in water-kerosene baths. Evidently the energy consumed for formation and maintaining the droplets increases by gas flow rate and oil height. The slope of the lines increases with oil height, which shows that the dependence of interfacial energy on gas flow rate becomes more pronounced at larger oil heights.

Figure 12 shows the interfacial energy against gas flow rate for different silicone oil thicknesses. As mentioned for water-kerosene baths, the energy consumed for formation and maintaining the droplets increases by gas flow rate and oil height and also the dependence of interfacial energy on gas flow rate becomes stronger at larger oil heights. As expected, an abrupt change appears in water-silicone oil

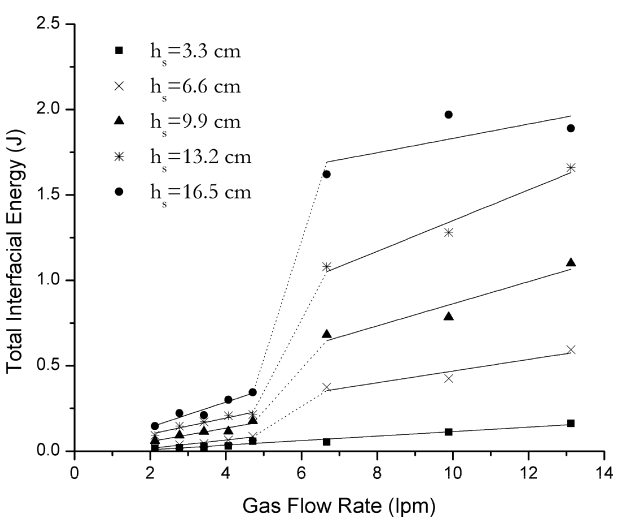

Fig. 12. Interfacial energy associated with the emulsified region at the presence of silicone oil as upper phase.

baths at the same critical gas flow rate for interfacial area.

\section{Conclusions}

(1) The droplets size distribution in the emulsified region of water-silicone oil baths is wider than that of water-kerosene baths. Significant increase in the interfacial area up to two orders of magnitude, was observed under the experimental conditions.

(2) Sauter mean diameter of the droplets in the emulsified region of water-silicone oil bath is greater than that of water-kerosene baths. The droplets become smaller at higher gas flow rate and eventually become stable at a specific size.

(3) Interfacial area created as a result of inverse emulsification increases with gas flow rate and oil height.

(4) An abrupt increase in interfacial area occurs as a result of breaking the entire oil layer into small droplets. This happens to appear at a critical gas flow rate of 4.71 lpm for water-silicone oil baths.

(5) Energy dissipation associated with emulsification is composed of two components; the surface energy of the droplets or deformed interfaces, and the potential energy required for keeping the increased surface in its position. The former has a larger share of the total energy. Under the experimental conditions of this work, 81-93\% and 53-95\% of interfacial energy is used for detachment of the droplets, for water-kerosene and water-silicone oil systems respectively.

\section{REFERENCES}

1) M. Matsuo, C. Saito, H. Katayama, H. Hirata and Y. Ogawa: Tetsuto-Hagané, 76 (1990), 1879.

2) W. O. Philbrook and L. D. Kirkbride: J. Met., 8 (1956), 351.

3) W. F. Holbrook and T. L. Joseph: Trans. AIME, 120 (1936), 99.

4) A. Zaidi and H. Y. Sohn: ISIJ Int., 35 (1995), 234.

5) P. Dayal, K. Beskow, J. Bjorkvall and Du Sichen: Ironmaking Steelmaking, 33 (2006), 454.

6) Z. Lin and R. I. L. Guthrie: Metall. Trans., 25B (1994), 855

7) K. Beskow, P. Dayal, J. Bjorkvall and Du Sichen: Ironmaking Steelmaking, 33 (2006), 76.

8) Subagyo, G. A. Brooks, K. S. Coley and G. A. Irons: ISIJ Int., 43 (2003), 983.

9) Z. Han and L. Holappa: ISIJ Int., 43 (2003), 292.

10) G. A. Brooks, Y. Pan, Subagyo and K. S. Coley: Metall. Trans., 36B (2005), 525.

11) G. Akdogan and R. H. Eric: J. S. Afr. Inst. Min. Met., 35 (2004), 
589.

12) J. E. Olsen, H. Laux and J. B. Oian: Proc. of 5th Int. Conf. on CFD in the Process Industries, Melbourne, Australia, (2006).

13) D. Mazumdar, H. Nakajima and R.I. L. Guthrie: Metall. Trans., 19B (1988), 507.

14) S. Tanaka and R. I. L. Guthrie: Proc. of 6th Process Technologies Conf., Washington, DC, (1986)

15) D. Poggi, R. Minto and W. G. Davenport: J. Met., 21 (1969), 40.

16) D. Minto and W. G. Davenport: Trans. Inst. Min. Metall., 81 (1972), 36.

17) O. J. Ilegbusi, M. Iguchi and W. E. Wahnsiedler: Mathematical and Physical Modeling of Materials Processing Operations, Chapman \& Hall/CRC, Florida, (1999), 351

18) K. Krishnapisharody: Ph.D. Thesis, McMaster Univ., (2007).

19) O. Haida, T. Emi, S. Yamada and F. Sudo: Scaninject II, \# 20, MEFOS and Jernkontoret, Lulea, Sweden, (1980).

20) Q. Ying, L. Yun and L. Liu: Scaninject II, \# 21, MEFOS and Jernkontoret, Lulea, Sweden, (1980).

21) M. G. Frohberg, F. Gerlach and G. Handschuh: Steel Res., 61 (1990), 151.

22) S. H. Kim and R. J. Fruehan: Metall. Trans. , 18B (1987), 381.

23) S. H. Kim, R. J. Fruehan and R. I. L. Guthrie: Proc. of Steelmaking Conf., The Iron and Steel Society Inc., Pittsburgh, (1987), 107.

24) J. M. Chou, M. C. Chuang, M. H. Yeh, W. S. Hwang, S. H. Liu, S. T.
Tsai and H.S. Wang: Ironmaking Steelmaking, 30 (2003), 195.

25) S. Taniguchi, Y. Okada, A. Sasaki and A. Kikuchi: Proc. of 6th Int. Iron Steel Cong. ISIJ, Tokyo, (1990).

26) J. Mietz, S. Schneider and F. Oeters: Steel Res., 62 (1991), 1.

27) W. F. Porter, F. D. Richardson and K. N. Subramanian: Heat and Mass Transfer in Process Metallurgy, Inst. Mining Met., London, (1979), 79.

28) K. N. Subramanian and F. D. Richardson: J. Iron Steel Inst. Jpn., 206 (1968), 576.

29) J. K. Brimacombe and F. D. Richardson: Trans. Inst. Min. Metall., 80 (1971), C140.

30) F. D. Richardson, D. G. C. Robertson and B. B. Staples: Proc. of Physical Chemistry in Metallurgy: The Darken Conf., U.S., Pittsburgh, (1976), 25.

31) J. K. Brimacombe and F. D. Richardson: Trans. Inst. Min. Metall., 82 (1973), C63.

32) P. Patel, M. G. Frohberg and K. Biswas: Proc. of Conf. Kinetics Metall. Process. Steelmaking, (1975) 180.

33) S. Asai, M. Kawachi and I. Muchi: Proc. of Conf. Foundry Process., (1988), 261.

34) H. Y. Sohn, D. G. C. Robertson, A. K. Agrawal and K. M. Iyer: Proc. of Int. Symp. on Bath Smelting, Warrendale, PA, (1992), 377.

35 D. R. Lide: CRC Handbook of Chemistry and Physics, CRC Press, Florida, (2000), 2. 